\title{
Hematoma disecante de esófago como diagnóstico diferencial del dolor torácico agudo
}

\section{Dissecting hematoma of the esophagus as a differential diagnosis of acute chest pain}

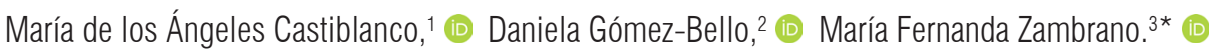

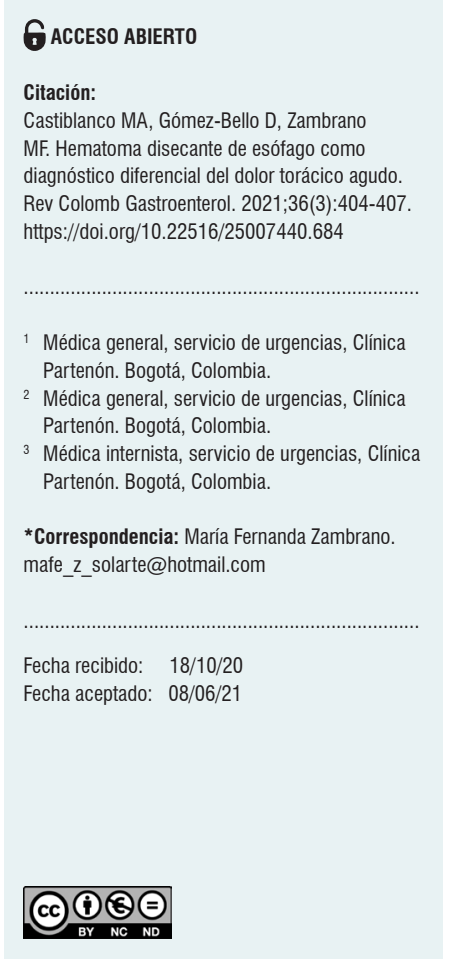

\begin{abstract}
Resumen
El dolor torácico es un motivo de consulta muy frecuente en el servicio de urgencias. El abordaje adecuado del paciente en el que se incluye el diligenciamiento de la historia clínica, el examen físico y la toma de paraclínicos iniciales son cruciales para determinar cuáles son los posibles diagnósticos diferenciales. A continuación, presentamos el caso de un paciente de 71 años que ingresa al servicio de urgencias por un dolor retroesternal intenso posterior al consumo de alimentos, en quien, ante la persistencia del dolor, biomarcadores cardíacos negativos y patología aórtica descartada, se realiza una endoscopia que evidencia un hematoma disecante del esófago. En general, el pronóstico es bueno, pero es de gran importancia una buena historia clínica para su sospecha.
\end{abstract}

\section{Palabras claves}

Hematoma intramural esofágico, infarto agudo de miocardio, arteria coronaria derecha, electrocardiograma, endoscopia de vías digestivas altas, tomografía computarizada, resonancia magnética.

\begin{abstract}
Chest pain is a very common reason for consultation in the emergency department. An adequate approach to the patient, including medical history, physical examination and initial laboratory tests, is crucial for determining possible differential diagnoses. The following is the case of a 71-year-old patient that visited the emergency room due to severe retrosternal pain after food consumption. In view of the persistence of pain and negative cardiac biomarkers, and after ruling out aortic disease, the patient underwent an endoscopy that showed dissecting hematoma of the esophagus. The prognosis is generally favorable, but a thorough medical history is required to suspect it.
\end{abstract}

\section{Keywords}

Intramural hematoma of the esophagus (IHE); Acute myocardial infarction; Right coronary artery; Electrocardiogram; Upper gastrointestinal endoscopy; Computed tomography; Magnetic resonance imaging.

\section{INTRODUCCIÓN}

El hematoma intramural esofágico (HIE) es una causa poco común de dolor torácico, en la que se presenta disección de la submucosa. Puede desencadenarse por alteraciones de la presión esofágica, traumas o espontáneamente. Su pre- sentación clínica se manifiesta con dolor torácico, disfagia/ odinofagia y hematemesis. Las ayudas imagenológicas permiten tanto su diagnóstico como la posibilidad de descartar diferenciales. En la mayoría de los casos, su pronóstico es bueno. A continuación, presentamos un caso clínico de dolor torácico secundario a HIE. 


\section{PRESENTACIÓN DEL CASO}

Un paciente de 71 años con antecedentes de hipertensión arterial, diabetes mellitus tipo 2 insulinorrequiriente, hipotiroidismo e infarto agudo de miocardio (IAM) con obstrucción de la arteria coronaria derecha (ACD) del $20 \%$ el año previo, consulta al servicio de urgencias de la Clínica Partenón en la ciudad de Bogotá, Colombia, por cuadro clínico de 40 minutos de evolución consistente en dolor retroesternal de inicio súbito, in crescendo, de intensidad 10/10, opresivo, irradiado a la región dorsal y mandíbula, asociado con un único episodio emético, diaforesis y sensación de náusea. Este dolor se presenta posterior a la ingesta alimentaria.

Dada la alta sospecha de un nuevo evento coronario, es trasladado a la sala de reanimación. Los signos vitales iniciales son: presión arterial: 171/100 mm Hg, frecuencia cardíaca (FC): 80 latidos por minutos (lpm), frecuencia respiratoria (FR): 18 (respiraciones por minuto (rpm), temperatura $(\mathrm{T}): 36^{\circ} \mathrm{C}$ y saturación de oxígeno $\left(\mathrm{SatO}_{2}\right)$ : $93 \%$. No requiere oxígeno suplementario; está álgido, diaforético y sus demás exámenes físicos son normales.

De forma inmediata se toma un electrocardiograma (ECG), el cual se encuentra en ritmo sinusal, inversión de la onda T en V1. En los paraclínicos iniciales se reporta: hemograma, función renal, electrolitos, tiempos de coagulación y perfil hepático normales, y biomarcador cardíaco negativo. Debido al corto tiempo entre el inicio de los síntomas y la consulta al servicio de urgencias, se realiza un nuevo control de troponina a las 3 horas, el cual fue también negativo, por lo que se descartó un evento coronario agudo.
Posteriormente, por la localización e intensidad del dolor se sospechó de una posible disección aórtica, para lo que se realiza una angio-TAC (tomografía axial computarizada) de tórax, que resulta negativa para alteraciones vasculares (Figura 1).

En búsqueda de diagnósticos diferenciales y ante un cuadro que inicia posterior a la ingesta alimentaria, se solicita una endoscopia de vías digestivas altas (EVDA), obteniendo como hallazgo un hematoma espontáneo disecante de la pared esofágica (Figura 2A y B).

Se continúa la hospitalización en la sala de reanimación para vigilancia clínica, hidratación intravenosa y la administración de un inhibidor de la bomba de protones. El paciente es valorado por el servicio de cirugía de tórax, quienes descartan la presencia de un cuerpo extraño intraluminal y mediastinitis.

Por estabilidad clínica, mejoría del dolor y adecuada tolerancia oral, se continúa la vigilancia en la sala de observación. Posteriormente se hospitaliza, se continúa con la administración intravenosa de un inhibidor de la bomba de protones, se realiza un hemograma de control sin evidencia de leucocitosis, neutrofilia, anemia ni trombocitopenia. Dada la evolución adecuada, se da egreso con signos de alarma, recomendaciones generales y cita de control ambulatoria por gastroenterología.

\section{DISCUSIÓN}

El HIE es una patología poco frecuente que se caracteriza por la presencia de colecciones de sangre en la pared esofágica $^{(1)}$. Respecto a su epidemiología, se ha observado que
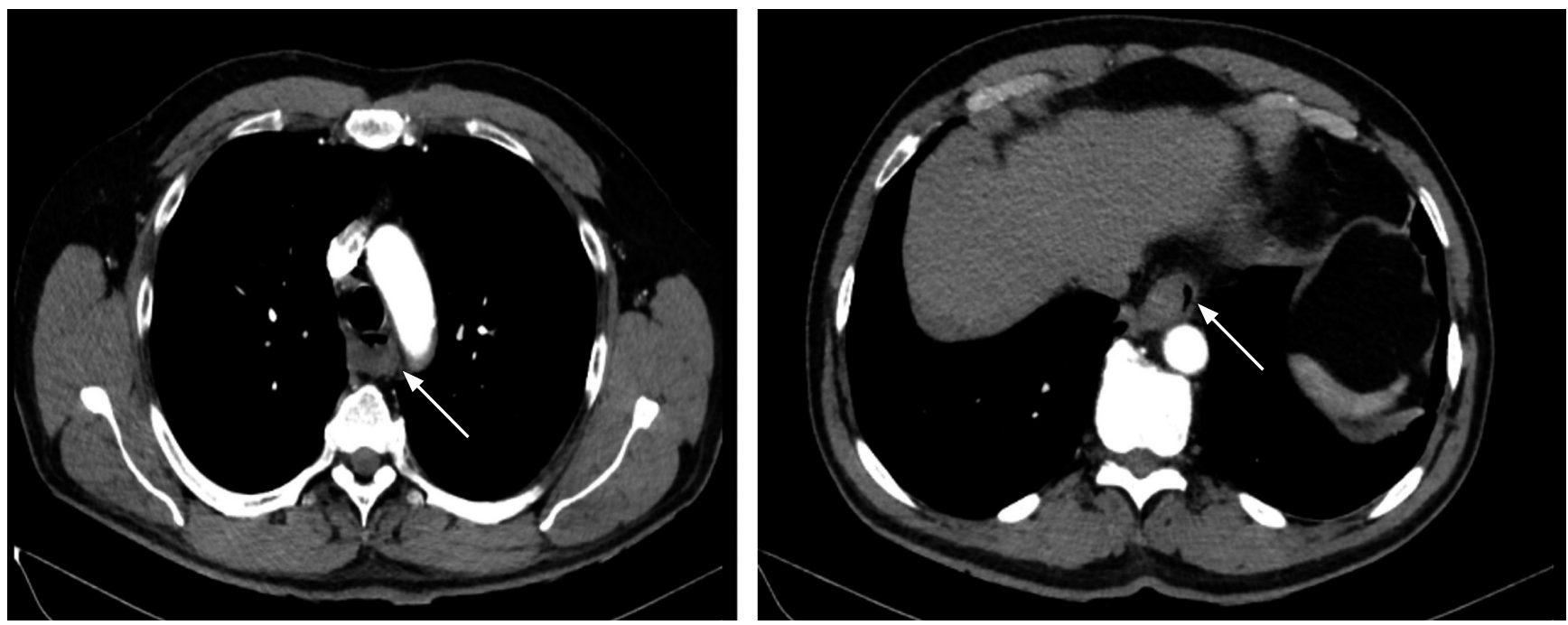

Figura 1. Angio-TAC de tórax: la flecha blanca señala un hematoma a nivel del esófago. 

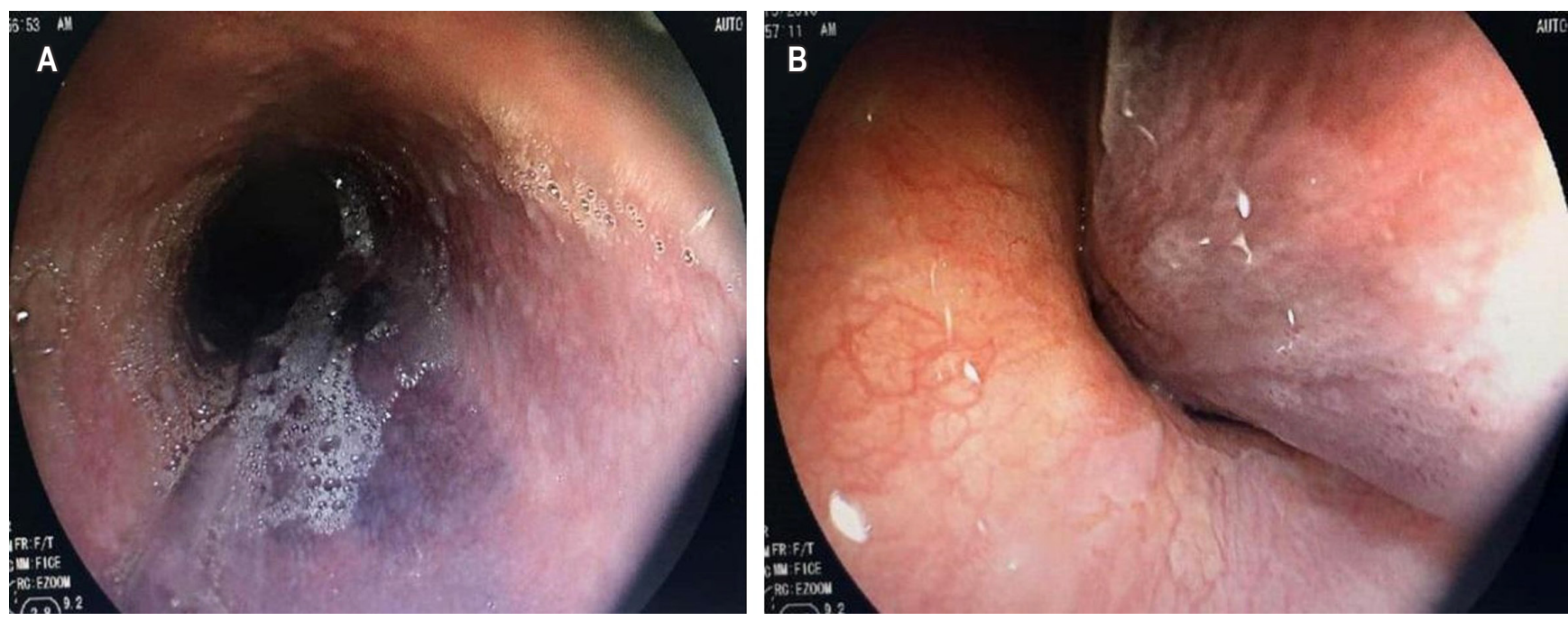

Figura 2. A. EVDA: esófago-hematoma; B. Hematoma de la pared lateral izquierda que se extiende hasta el cardias, condicionando la compresión intraluminal.

es más frecuente en la población femenina (riesgo relativo de 1,8$)$, con un promedio de edad de 63 años; se presenta una distribución bimodal con picos de edad a los 45 y a los 70 años ${ }^{(2-6)}$.

Las manifestaciones clínicas típicas incluyen dolor torácico, odinofagia/disfagia y hematemesis leve $\mathrm{e}^{(2,5,7)}$. El dolor torácico, presente en la mayoría de los pacientes (84 \%) y considerado el síntoma predominante, se caracteriza por ser de origen súbito, retroesternal y en ocasiones irradiado a la región dorsal o interescapular, como se manifestó en este paciente.

Por la forma de presentación de dicho dolor en la población adulta, se puede confundir como un síndrome coronario agudo, con el riesgo de empeorar el tamaño del hematoma y el cuadro gastrointestinal en caso de iniciar un manejo antiisquémico o fibrinolítico ${ }^{(4)}$. En ese sentido, una historia clínica detallada ayuda en el diferencial de dolor torácico en estos pacientes, que incluye disección aórtica, síndromes coronarios o perforación esofágica.

La hematemesis se presenta en menor frecuencia (56\%), secundaria a la disección del hematoma por la mucosa esofágica $^{(2)}$. Solo el $33 \%$ de los pacientes presentan la tríada clásica y a un $90 \%$ se les manifiesta con un síntoma, como en el caso presentado, que inició solo con dolor torácico. Una vez sospechada la patología esofágica, se deben considerar como diferenciales el síndrome de Mallory-Weiss y el síndrome de Boerhaave (ruptura completa de la pared esofágica $)^{(6)}$.

Dentro de los factores desencadenantes se encuentran ${ }^{(3)}$ :
- el aumento súbito de la presión esofágica, que se puede presentar con maniobras de Valsalva, náuseas o emesis; - secundario a procedimientos endoscópicos;

- de forma espontánea.

En los casos idiopáticos, se ha observado predisposición por alteraciones esofágicas (por ejemplo, acalasia) o de la coagulación (antiagregación o anticoagulación). Inclusive, se han presentado casos de disección esofágica postrombólisis en síndromes coronarios con elevación del segmento $\mathrm{ST}^{(4,8)}$.

Ante la sospecha clínica, los paraclínicos como el electrocardiograma y la radiografía de tórax se pueden realizar para descartar diferenciales. Tradicionalmente, se realizaba un esofagograma que evidenciaba un defecto de llenado o "signo de doble barril" en el tercio distal esofágico (sitio de mayor afectación por presentar menor soporte de estructuras contiguas) $)^{(2,6)}$.

Actualmente, la tomografía axial computarizada/resonancia magnética nuclear (TAC/RMN) de tórax permite llegar de forma más rápida y menos invasiva al diagnóstico, excluyendo a su vez diferenciales como disección aórtica o perforaciones. Lo que se evidencia en las imágenes es un engrosamiento esofágico bien definido y sin realce ${ }^{(6)}$. Por último, la EVDA permite esclarecer la localización del hematoma en la submucosa y usualmente se observa como una lesión violácea o azul en la región posterior, que en ocasiones puede confundirse con várices o tumores ${ }^{(2)}$.

Una vez diagnosticado, el tratamiento es principalmente conservador con líquidos intravenosos y progresión de la 
dieta según la evolución del paciente. La cirugía solamente está indicada en casos de sangrado abundante, perforación esofágica o formación de absceso complicado de difícil drenaje $^{(1,3)}$. Los protectores gástricos no han demostrado beneficio alguno sobre la patología ${ }^{(2)}$. Aproximadamente, el $75 \%$ de los pacientes presentan una recuperación completa entre los 7-14 días, sin complicaciones, siendo los casos de mal pronóstico relacionados con pacientes terminales o con acalasia (mayor recurrencia de resangrado) ${ }^{(2)}$. El seguimiento de estos pacientes se puede realizar por EVDA o ecoendoscopia.

\section{CONCLUSIONES}

En conclusión, el HIE es una patología poco frecuente que debe considerarse en pacientes con dolor torácico de inicio súbito con síntomas gastrointestinales como disfagia o hematemesis. Su sospecha se debe basar principalmente en una buena historia clínica, considerando los riesgos de complicaciones con un mal enfoque del paciente (por ejemplo, inicio de antiagregación/anticoagulación por sospecha de síndrome coronario).

\section{REFERENCIAS}

1. Amott DH, Wright GM. Dissecting haematoma of the oesophagus masquerading as acute myocardial infarction. Med J Aust. 2006; 184(4):182-3. https://doi. org/10.5694/j.1326-5377.2006.tb00182.x

2. Cao DT, Reny JL, Lanthier N, Frossard JL. Intramural hematoma of the esophagus. Case Rep Gastroenterol. 2012;6(2):510-7. https://doi.org/10.1159/000341808

3. Cullen SN, McIntyre AS. Dissecting intramural haematoma of the oesophagus. Eur J Gastroenterol Hepatol. 2000;12(10):1151-62. https://doi. org/10.1097/00042737-200012100-00014

4. Huddy J, Kotecha A, Dussek J, McNair A. Dissecting intramural hematoma of the esophagus after thrombolysis for myocardial infarction. Gastrointest Endosc. 2005;61(2):340-3. https://doi.org/10.1016/S00165107(04)02575-1

5. Kimmoun A, Abboud G, Steinbach G, Tellaroli JC, Bemer M. Hématome intramural disséquant de l'oesophage: une cause rare de douleur thoracique [Dissecting intramural hematoma of the esophagus: a rare cause of chest pain]. Presse Med. 2008;37(3 Pt 1):420-3. https://doi. org/10.1016/j.lpm.2007.04.043

6. Modi P, Edwards A, Fox B, Rahamim J. Dissecting intramural haematoma of the oesophagus. Eur J Cardiothorac Surg. 2005;27(1):171-3. https://doi.org/10.1016/j. ejcts.2004.09.012

7. Sen A, Lea RE. Spontaneous oesophageal haematoma: a review of the difficult diagnosis. Ann R Coll Surg Engl. 1993;75(4):293-5.

8. Silva MJ, Saiote J, Salvado V, Nunes AP, Duarte P. Intramural hematoma of the esophagus after thrombolysis for ischemic stroke. Am J Emerg Med. 2015;33(3):481. e1-2. https://doi.org/10.1016/j.ajem.2014.08.069 